\title{
Pharmacological treatment of idiopathic pulmonary fibrosis: from the past to the future
}

\author{
Katerina M. Antoniou, George A. Margaritopoulos and Nikos M. Siafakas
}

Affiliations: Interstitial Lung Disease Unit, University Hospital of Heraklion, Medical School, University of Crete, Heraklion, Greece.

Correspondence: K.M. Antoniou, Dept of Thoracic Medicine, University of Crete, Medical School, 71110 Heraklion, Crete, Greece. E-mail: kantonioulamed.uoc.gr

ABSTRACT During the past decade important progress has been made regarding the pathogenesis of idiopathic pulmonary fibrosis (IPF), which is the most devastating form of idiopathic interstitial pneumonia with a median survival of 3 years. The knowledge gained has been used to design multicentre, randomised, placebo-controlled trials in order to investigate agents with different mechanisms of action. Encouraging results have led to licensing of the first IPF-specific drug, pirfenidone. However, the road to successful treatment is still long. The main aim for the future should be the careful design of clinical trials, by choosing the most clinically meaningful end-point and keeping in mind that combination of various agents may be more effective. This approach has been used in the treatment of lung cancer with which IPF presents many similarities.

0 @ERSpublications

Progress toward the treatment of IPF and aims for future clinical trials http://ow.ly/mPUqb

\begin{abstract}
Introduction
Idiopathic pulmonary fibrosis (IPF) is the most frequent and devastating form of idiopathic interstitial pneumonia [1]. The median survival from the time of diagnosis is 3 years and this explains why IPF is currently considered more lethal than many cancers [2]. The incidence and prevalence of IPF varies between studies. The true incidence and prevalence of IPF is not well established, due to the lack of a uniform definition of IPF in older studies which provided quantitative data on IPF incidence and/or prevalence, a lack of diagnostic criteria, differences in case finding methodologies and study designs. Hence, the prevalence and annual incidence of IPF in the USA varies from 14 to 27.9 cases per 100000 population and 6.8-8.8 per 100000 population, respectively, using narrow case definitions, and from 42.7 to 63 cases per 100,000 population and 16.3-17.4 per 100000 population, respectively, using broad case definitions [3]. In Europe, the prevalence and annual incidence ranges from 1.25 to 23.4 cases per 100000 population and 0.22-7.4 per 100000 population, respectively [3].
\end{abstract}

According to the recent official American Thoracic Society(ATS)/European Respiratory Society (ERS)/ Japanese Respiratory Society/Latin American Thoracic Association statement the diagnosis of IPF requires: 1) exclusion of other known causes of interstitial lung disease (ILD) (e.g. domestic and occupational environmental exposures, connective tissue disease, and drug toxicity); 2) the presence of an usual interstitial pneumonia (UIP) pattern on high-resolution computed tomography in patients not subjected to surgical lung biopsy; 3) specific combinations of high-resolution computed tomography and surgical lung biopsy patterns in patients subjected to surgical lung biopsy [1,4]. The multidisciplinary team, involving

Received: April 032013 | Accepted after revision: May 132013

Conflict of interest: None declared.

Provenance: Publication of this peer-reviewed article was supported by the World Scleroderma Foundation, Switzerland (principal sponsor, European Respiratory Review issue 129).

Copyright @ERS 2013 
pulmonologists, radiologists and pathologists with a special interest in the field of ILDs, represents the "gold standard" diagnostic approach [5].

Remarkable progress has been achieved over the past decade regarding the pathogenesis of this dismal disease $[6,7]$. Originally, IPF was considered an inflammation-driven process. However, mainly due to the lack of efficacy of the anti-inflammatory treatment approach, there was a shift to the current view that IPF is an epithelial-driven and fibroblast-activated process, in which inflammation is a secondary event [8]. Injurious environmental agents, such as viral infections, gastro-oesophageal reflux and smoking [9-12], acting in a background of genetic predisposition may initiate the cascade of the events that lead to simultaneous activation of multiple pathogenetic pathways, resulting in activation of myofibroblasts, exaggerated deposition of extracellular matrix and consequently the development of IPF [13]. In the normal lung injuries are repaired with restitutio ad integrum, but in the IPF lung the epithelium fails to restore itself. Various mechanisms, such as accelerated senescence due to premature telomere shortening and adaption of a "senescence associated secretory phenotype" with production of profibrotic mediators, surfactant abnormalities, and endoplasmic reticulum stress, lead to exhaustion of the alveolar epithelial precursor cells with consequent defective re-epithelialisation [14-19]. Despite this progress the underpinning pathogenetic mechanisms of IPF are not fully understood and, to date, no definite drug for this disease is available, underlining the gap between pathogenetic comprehension and pharmacological studies. However, the knowledge achieved has provided the basis for an increasing number of clinical trials targeting both cellular and molecular pathways implicated in the pathogenesis of IPF (table 1). 114 patients participated in the four clinical trials published from 1989 to 1999 , whereas from 2000 to 2010 there were 2861 patients recruited into 11 studies $[34,35]$.

\section{Completed clinical trials}

\section{Corticosteroids, azathioprine and $\mathrm{N}$-acetyl-cysteine}

In 2000, the ATS/ERS statement on IPF recommended the use of prednisone and azathioprine for the treatment of IPF [36], based on a randomised, double-blind, placebo-controlled study in which the use of the combined therapy with prednisone and azathioprine was shown to have a marginal effect on survival, compared with prednisone and placebo, without any effect on lung function [37]. In the years that followed, the change of view regarding the pathogenesis of IPF, coupled with the lack of efficacy of this approach, led to a strong negative recommendation for both prednisone monotherapy and combination therapy in the recent guidelines [1]. No placebo-controlled trials were identified in a recent meta-analysis to support the use of steroids in IPF $[38,39]$.

$\mathrm{N}$-acetyl-cysteine (NAC), a precursor of the antioxidant glutathione, stimulates glutathione synthesis, increases intra- and extracellular levels and, thus, partially restores glutathione levels [40, 41]. The IFIGENIA trial was a multicentre, double-blind, randomised, placebo-controlled phase III trial which evaluated the effect of a high dose of oral NAC (i.e. $600 \mathrm{mg}$ three times daily) together with low dose of

TABLE 1 Completed clinical trials in idiopathic pulmonary fibrosis

Drug Mechanism of Action

\section{IFIGENIA [20]}

PANTHER-IPF [21]

TANIGUCHI [22]
CAPACITY 1 [23]
CAPACITY 2 [23]
ACE-IPF [24]
TOMORROW [25]
DANIELS [26]
STEP-IPF [27]
BUILD-1 [28]
BUILD-3 [29]
ARTEMIS-IPF [30]
MUSIC-IPF [31]
RAGHU [32]
INSPIRE [33]

TANIGUCHI [22]

CAPACITY 2 [23]

ACE-IPF [24]

TOMORROW [25]

DANIELS [26]

STEP-IPF [27]

BUILD-1 [28]

BUILD-3 [29]

MUSIC-IPF [31]

INSPIRE [33]

NAC
Prednisone
Azathioprine
NAC
Pirfenidone
Pirfenidone
Pirfenidone
Warfarin
BIBF 1120
matinib mesylate
Sildenafil
Bosentan
Bosentan
Ambrisentan
Macitentan
IFN- $\gamma$
IFN- $\gamma$

Antioxidant

Antioxidant

Immunosuppression

Antifibrotic

Antifibrotic

Antifibrotic

Anticoagulant

Tyrosine-kinase inhibitor

Tyrosine-kinase inhibitor

Phosphodiesterase-5 inhibitor

Endothelin-receptor antagonist

Endothelin-receptor antagonist

Endothelin-receptor antagonist

Endothelin-receptor antagonist Immunomodulation Immunomodulation

NAC: $\mathrm{N}$-acetyl-cysteine; IFN: interferon. 
prednisone and azathioprine compared with prednisone and azathioprine alone [20]. The primary endpoint was the change in vital capacity (VC) and diffusing capacity of the lung for carbon monoxide (DLCO) at 12 months. A significant reduction in the decline of both VC and DLCO was shown, whereas there was no effect on the survival between the two groups. However, this trial had not been powered to assess the effect of the treatment on survival. The trial raised a lot of criticism due to the high drop-out rate, which may have biased the final conclusion, and mainly because of the lack of a true placebo arm which made it difficult to draw firm conclusions about the real effect of NAC. Questions have been raised as to whether the observed result was due to the effect of NAC or due to the interaction between drugs with different mechanisms of action. In order to shed light on this matter a multicentre, double-blind, randomised, placebo-controlled trial, named PANTHER-IPF, has been designed [21]. The patients were assigned to one of the three arms: 1) prednisone, azathioprine and NAC; 2) NAC alone; and 3) placebo. The primary outcome was the change in forced vital capacity (FVC) at 60 weeks. On October 2011, a press release announced that the triple therapy arm had been discontinued due to an excess number of deaths (11\% versus $1 \%$ ), hospitalisations ( $29 \%$ versus $8 \%$ ) and higher prevalence of adverse effects (31\% versus $9 \%$ ) compared with the placebo arm [42]. No safety issues have been raised for the NAC monotherapy arm and the trial is still ongoing for the NAC and placebo arms. However, some issues concerning these results should be discussed. Firstly, these events happened during the first 15 weeks of the trial, when the dose of prednisone was high. At the beginning of the trial the dose of prednisone was $0.5 \mathrm{mg} \cdot \mathrm{kg}^{-1}$ of ideal body weight and was tapered to $0.15 \mathrm{mg} \cdot \mathrm{kg}^{-1}$ over a 25 week period. Secondly, almost half of the patients enrolled in the trial were aged $>70$ years and this group of patients are more susceptible to respiratory infections due to the immunosuppressive effect of azathioprine. Thus, pending further analysis a recent European Respiratory Journal editorial anticipates that most patients and physicians will decide against starting immunosuppressive therapy de novo in IPF [43]. It has been also stated that patients with definite IPF treated with triple therapy for $>12$ months, without evidence of disease progression or adverse effects due to the treatment may continue on triple therapy after detailed discussion with their clinicians. In patients with definite IPF treated with triple therapy for $<12$ months, clinical decisions should be reached after carefully balancing the risks and benefits. In patients with definite IPF who have experienced infections as a side-effect of the immunosuppression the triple therapy should be discontinued.

\section{Pirfenidone}

Pirfenidone is an orally administered drug with antifibrotic, anti-inflammatory and antioxidant properties [44]. Results from experimental models [45-49] have led to a phase II multicentre, randomised, placebocontrolled trial in which patients have been assigned to receive $600 \mathrm{mg}$ of pirfenidone three times daily or placebo for 12 months [50]. The primary end-point was change in the lowest arterial oxygen saturation measured by pulse oximetry during the 6-min walk test. However, given an excess of acute exacerbations in the placebo group compared with the pirfenidone arm the trial was terminated after 9 months. Although the primary end-point was not met, a significantly slower decline of FVC was observed in the treatment arm. Based on these results, a multicentre, double-blind, randomised, placebo-controlled phase III trial has been designed, in Japan, in order to test the efficacy and safety of the drug over 52 weeks [22]. The primary end-point was the change in FVC from baseline to 52 weeks and secondary end-points included progression-free survival, defined as the time until the first progression event (decline in FVC $>10 \%$, or death), and change in lowest arterial oxygen saturation measured by pulse oximetry during the 6-min steady-state exercise test. The patients have been assigned to receive a high dose $\left(1800 \mathrm{mg} \cdot \mathrm{day}^{-1}\right)$ or a low dose of pirfenidone $\left(1200 \mathrm{mg} \cdot \mathrm{day}^{-1}\right)$ or placebo in the ratio of $2: 1: 2$. The primary end-point has been met, as there was a statistically significant difference in the deterioration of FVC between the placebo arm $(-0.16 \mathrm{~L})$ and the high-dose $\operatorname{arm}(-0.09 \mathrm{~L})(\mathrm{p}=0.0416)$. With regards to the secondary end-points, the progression-free survival was significantly longer in the high-dose arm compared with the placebo arm $(p=0.0280)$. The major side-effect was photosensitivity, which presented almost equally in the two treatment arms. Following this study the drug was licensed in Japan, in 2008. Although successful, the study has received a lot of criticism, because the initial end-point (i.e. change in lowest arterial oxygen saturation measured by pulse oximetry during the 6-min steady-state exercise test) has been changed even if this has been carried out before unblinding the study, and because of the statistical analysis used to handle the missing values.

Two similar trials, known as the CAPACITY trials (studies 004 and 006), have been carried out in North America, Australia and Europe for 72 weeks [23]. Patients with mild-to-moderate IPF were screened for eligibility using the following functional criteria: FVC $\geqslant 50 \%$ predicted; DLCO $\geqslant 35 \%$ predicted; and 6-min walk test $(6 \mathrm{MWT})$ distance $\geqslant 150 \mathrm{~m}$. In study 004 the patients were assigned to receive either high-dose pirfenidone $\left(2403 \mathrm{mg} \cdot \mathrm{day}^{-1}\right)$ or low-dose pirfenidone $\left(1197 \mathrm{mg} \cdot \mathrm{day}^{-1}\right)$ or placebo; whereas in study 006 the patients were assigned to receive high-dose pirfenidone $\left(2403 \mathrm{mg} \cdot \mathrm{day}^{-1}\right)$ or placebo. In both studies the primary end-point was the change of FVC \% pred from baseline to week 72. 
Only study 004 met the primary end-point, as there was a significant reduction in FVC decline at week 72 in the high-dose group compared with placebo $(\mathrm{p}<0.001)$. Pooled analysis of primary end-point data from both studies also showed that pirfenidone significantly reduced the decline of FVC \% pred compared to placebo $(p<0.005)$. Pooled analysis of secondary end-points demonstrated a $30 \%$ reduction in the percentage of patients with a categorical decline in FVC $\geqslant 10 \%$ at week $72(\mathrm{p}=0.003)$, a $31 \%$ reduction in the mean decline in 6MWT distance $(\mathrm{p}>0.001)$ and a $26 \%$ reduction in the risk of death or disease progression (hazard ratio (HR) $0.74,95 \%$ CI $0.57-0.96 ; \mathrm{p}=0.025$ ). Pirfenidone was generally well tolerated, with the most common side-effects being gastrointestinal discomfort and photosensitivity.

A recent Cochrane review, including the four studies mentioned above, has shown that treatment with pirfenidone reduced the risk for disease progression by $30 \%$ (HR 0.70, 95\% CI 0.56-0.88) [39]. Based on the results of these studies, pirfenidone was licensed in Europe in 2011, for patients with mild-to-moderate disease. In USA, the lack of success of study 006 in reaching the primary end-point led the Food and Drug Administration to decline approval. Therefore, a double-blind, randomised, placebo-controlled phase III trial (ASCEND) is currently being conducted in US for a total of 52 weeks, in order to test the safety and efficacy of pirfenidone in IPF patients receiving $2403 \mathrm{mg} \cdot \mathrm{day}^{-1}$ versus placebo (NCT01366209). The results are expected in mid-2013.

An open-label, extension study (RECAP) has been designed to evaluate the long-term safety of high-dose pirfenidone (i.e. $2403 \mathrm{mg} \cdot \mathrm{day}^{-1}$ ) in patients with IPF. Patients eligible for this study were those who received $\geqslant 80 \%$ of the scheduled study drug doses and completed the week 72 final study visit in either Study 004 or Study 006. The majority of patients experienced only mild-to-moderate adverse events and relatively few patients discontinued treatment due to an adverse event. Adverse events, such as gastrointestinal symptoms and photosensitivity, were mild-to-moderate, transient, rarely led to treatment discontinuation and, importantly, were consistent with observations from the Phase III clinical trials. Hence, long-term treatment with pirfenidone is considered safe and generally well tolerated [51].

\section{Anticoagulants}

There is evidence suggesting that the microenvironment of the fibrotic lung is procoagulant and antifibrinolytic [52]. Coagulation factors X and VII are overexpressed in the fibrotic lung, where they induce myofibroblast differentiation and fibroblast proliferation by binding to proteinase-activated receptor-1 and -2 , respectively $[53,54]$. Moreover, the diagnosis of IPF is associated with current or past events of thromboembolism [55]. Based on these observations, it was thought that anticoagulation therapy may be beneficial for IPF. In 2005, KuBo et al. [56] published the data from a small prospective, randomised, controlled, open label study in which patients with IPF received either prednisolone or prednisolone plus oral warfarin in an outpatient setting, or prednisolone plus low-molecular weight heparin if admitted in the hospital because of an acute exacerbation. They observed a significantly lower mortality due to acute exacerbations in the anticoagulant group compared with controls. However, there were important limitations to the study: it was not blinded, lacked a placebo arm, included a small cohort of patients and there was selective withdrawal of patients from the anticoagulant arm.

The more recent ACE-IPF study, a double-blind, randomised, placebo-controlled trial, was set up to evaluate the efficacy and safety of warfarin in IPF [24]. The participants were randomised to receive either warfarin or placebo in a 1:1 ratio for a total of 48 weeks. However, when 145 of the planned 245 patients had been recruited and the mean follow-up time was 28 weeks, the study was prematurely terminated, due to an increase in mortality and low evidence of benefit in the warfarin group compared with placebo. The majority of deaths in the treatment arm were related to disease progression or acute exacerbation, whereas there were no deaths attributed to bleeding, the major complication of warfarin. The authors concluded that the activation of a vitamin $\mathrm{K}$ dependent pathway may be not beneficial and should not be a target for future therapeutic approaches in IPF. Although it has been proposed that the discrepancy between the results of the ACE-IPF study [24] and the one by KuBo et al. [56] could be attributed to differences in the genetic background of the study population and in the methodology of the two studies [57], the results of the ACEIPF study have placed, for the time being, a "strong no" label on the use of warfarin in IPF.

\section{Tyrosine kinase inhibitors}

BIBF 1120 is a triple inhibitor of tyrosine kinases receptors, such as platelet derived growth factor (PDGF), vascular endothelial growth factor and fibroblast growth factor receptors, which are implicated in the process of fibrogenesis [58]. Recently, the results of a double-blind, randomised, placebo-controlled phase II trial of the efficacy and safety of BIBF 1120 (TOMORROW) have been published [25]. 432 patients with IPF were assigned to receive four different doses of the drug or placebo for 12 months. The primary end-point was the annual rate of decline in FVC, and the secondary end-points included acute exacerbations, quality of life measures and total lung capacity (TLC). In subjects from the group receiving the highest dose of BIBF 
1120 (i.e. $150 \mathrm{mg}$ twice daily), the annual rate of decline of FVC was $0.06 \mathrm{~L}$ compared with $0.19 \mathrm{~L}$ in the placebo arm. Moreover, fewer acute exacerbations, a preserved quality of life, moderate gastrointestinal symptoms and liver toxicity were also observed in the high dose arm compared with placebo. These observations have led to the development of two larger multicentre phase III trials, which are currently being carried out using the annual rate of decline in FVC as the primary end-point (NCT01335477 and NCT01335464). The results of these studies are expected in early 2014.

Imatinib mesylate is another inhibitor of tyrosine kinases, with activity against PDGF receptors and the c-kit and $\mathrm{c}-\mathrm{ABL}$ tyrosine kinases. Based on the results of experimental models, showing that imatinib exerted a protective effect against the development of fibrosis [59-61], a phase II multicentre, double-blind, placebocontrolled trial of 119 patients with mild-to-moderate IPF was carried out for 96 weeks [26]. The primary end-point, defined as time to disease progression (10\% decline in FVC \% pred from baseline or death over a 96-week period), was not met and, moreover, neither was an effect on lung function observed. It was concluded that imatinib mesylate is not an effective therapy in patients with IPF.

\section{Pulmonary hypertension in IPF}

Sildenafil, a phosphodiesterase type 5 (PDE-5) inhibitor which causes selective pulmonary vasodilatation and does not alter ventilation/perfusion matching and arterial oxygenation, has been investigated in patients with end-stage IPF [27]. The STEP-IPF trial was a double-blind, randomised, placebo-controlled trial in which patients initially received either oral sildenafil $(20 \mathrm{mg}$ three times daily) or placebo for 12 weeks; subsequently, there was an open label evaluation of the patients receiving sildenafil for 12 weeks. The primary end-point of improvement in 6-min walking distance (6MWD) was not achieved. However, a number of secondary end-points were satisfied as there was an improvement in DLCO, transfer coefficient of the lung for carbon monoxide and arterial oxygenation which, in absence of a simultaneous effect on FVC, indicated a significant effect on the pulmonary vessels.

Bosentan, an endothelin-1 receptor antagonist effective in pulmonary arterial hypertension, was initially tested in small trials for IPF because of its antifibrotic properties and showed some encouraging results, which were later replaced by disappointing results from larger trials $[28,29]$. In BUILD-1, a randomised, placebo-controlled, phase II trial, the primary end-point of change in 6MWD was not met. However, a trend towards a delayed time to disease progression in favour of bosentan was observed and has been shown to be statistically significant in cases of "atypical" IPF appearance in high resolution computed tomography and hence diagnosed by surgical lung biopsy [28]. Subsequently, a larger double-blind, randomised, placebo-controlled trial (BUILD-3) explored the effect of bosentan on the time to IPF worsening or death, and no significant effects were observed when compared with placebo [29].

It has been hypothesised that ambrisentan, an endothelin A receptor antagonist, may reduce disease progression in patients with IPF. The ARTEMIS-IPF trial was a randomised, double-blind, placebocontrolled trial of ambrisentan with the time to IPF disease progression as the primary end-point. The study was terminated when $75 \%$ of the planned subjects had been enrolled and had a mean exposure of 34 weeks because of lack of evidence of a treatment benefit in the group of patients randomised to receive ambrisentan [30]. Thus, ambrisentan was ineffective in treating IPF and was associated with an increased risk of hospitalisation due to respiratory events.

Macitentan is an endothelin receptor antagonist. The MUSIC trial was a randomised, double-blind, placebo-controlled phase II trial in which macitentan at a dose of $10 \mathrm{mg} \cdot \mathrm{day}^{-1}$ was tested against placebo. The primary end-point of the change in FVC from baseline to month 12 was not met, although the drug was generally well tolerated [31].

\section{Interferon $\boldsymbol{\gamma}$}

Interferon (IFN) $-\gamma$ is a cytokine with immunomodulatory and antifibrotic properties. It has been observed in experimental models that IFN- $\gamma$ has inhibitory effects in different stages of the fibrogenic process [62-65]. Initial encouraging results from a small single-centre study [66] have not been replicated by two larger trials. RAGHU et al. [32] tested the use of subcutaneous IFN- $\gamma$ for 58 weeks in 330 patients with IPF compared with placebo, without showing any effect in terms of progression-free survival, pulmonary function or quality of life. Subsequently a larger trial (INSPIRE) involving $800 \mathrm{IPF}$ patients also failed to show any benefit in terms of mortality in favour of IFN- $\gamma[33]$.

\section{Tumour necrosis factor- $\alpha$ antagonists}

Tumour necrosis factor (TNF)- $\alpha$ is cytokine with profibrotic properties whose levels have been found to be elevated in both the lungs of experimental animal models and of patients with IPF. Etanercept is a soluble antagonist of TNF- $\alpha$ widely used in rheumatoid arthritis for its anti-inflammatory properties. 
A randomised, double-blind, placebo-controlled phase II trial explored the effect of etanercept on the change in FVC from baseline to week 48. Although the drug was well tolerated it failed to satisfy the primary end-point; however, some nonsignificant trends in DLCO and 6MWT were observed [67].

\section{Ongoing clinical trials}

A number of early phase clinical trials investigating new molecules are ongoing (table 2). Increased levels of interleukin (IL)-13, a cytokine secreted by T-helper type 2 cells, have been found in the lungs of IPF patients [68]. IL-13 is a stimulator of fibroblast proliferation and if overexpressed in animal models it induces pulmonary fibrosis; whereas if IL-13 is neutralised pulmonary fibrosis is attenuated [69]. A double-blind, placebo-controlled phase II trial investigating QAX576, a human anti-IL-13 monoclonal antibody, for 12 months is currently recruiting patients (NCT01266135). The primary end-point is the change in FVC at 52 weeks as compared with baseline.

Chemokine (C-C motif) ligand (CCL) 2 cytokine, also known as monocyte chemoattractant-1, regulates the recruitment of cells, such as monocytes, memory T-cells and dendritic cells, to the site of lung injury. It has been hypothesised that this molecule is implicated in the development of fibrosis as fibroblasts from IPF lungs show increase responsiveness to CCL2 and, moreover, when neutralised in the animal model of fibrosis collagen deposition was reduced [70]. CNTO 888, a monoclonal antibody against CCL2, has been investigated in a double-blind, placebo-controlled phase II trial (NCT00786201). The study has been completed and the results are awaited.

Connective tissue growth factor (CTGF) is a cytokine implicated in the development of fibrosis by contributing to myofibroblast activation and extracellular matrix deposition [71]. In an animal model of radiation-induced pulmonary fibrosis administration of FG-3019, a monoclonal antibody to CTGF, resulted in attenuation of the fibrotic process [71]. The safety of FG-3019 has been investigated in a phase I trial (NCT00074698) and, currently, a phase II trial is investigating the safety and tolerability of this antibody (NCT01262001).

Transforming growth factor (TGF)- $\beta 1$, an isoform of TGF- $\beta$, is an important mediator of pulmonary fibrosis that inhibits the proliferation of epithelial cells, promotes epithelial migration, stimulates the epithelial-mesenchymal transition and enhances epithelial apoptosis. In addition, it stimulates fibroblast proliferation and collagen production, and inhibits fibroblast apoptosis [5]. GC1008 is an antibody against TGF- $\beta$ and has been tested in a phase I trial in IPF patients (NCT00125385). The study has been completed and the results are awaited. A phase II multicentre, randomised, double-blind, placebo-controlled trial of STX-100, a monoclonal antibody against $\alpha_{\mathrm{v}} \beta 6$ integrin which activates TGF- $\beta$, is also being carried out (NCT01371305).

Excessive extracellular matrix deposition leading to distortion of the lung architecture is a critical step in the development of IPF. Lysyl oxidase and lysyl oxidase-like (LOXL) enzymes promote crosslinking of fibrillar collagen, which is a major component of extracellular matrix. Increased levels of LOXL2 have been found in IPF, as well as in liver fibrosis and in various types of cancer possibly participating in tumour invasion [72-74]. It has been proposed that inhibition of excessive extracellular matrix deposition may be a potential treatment target for IPF. In the light of the results showing that neutralisation of LOXL2 with a monoclonal antibody (AB0024) attenuated fibrosis in the bleomycin animal model [73], a phase I trial has been designed and completed, but the results have not yet been published (NCT01362231).

\section{TABLE 2 Ongoing clinical trials in idiopathic pulmonary fibrosis}

Trial $^{\# \quad \text { Drug Mechanism of action }}$

$\begin{array}{lll}\text { ASCEND (NCT01366209) } & \text { Pirfenidone } & \text { Antifibrotic } \\ \text { NCT01335477 } & \text { BIBF 1120 } & \text { Tyrosine-kinase inhibitor } \\ \text { NCT01335464 } & \text { BIBF 1120 } & \text { Tyrosine-kinase inhibitor } \\ \text { NCT01266135 } & \text { QAX576 } & \text { Anti-IL-13 monoclonal antibody } \\ \text { NCT00786201 } & \text { CNTO 888 } & \text { Anti-CCL-2 monoclonal antibody } \\ \text { NCT01262001 } & \text { FG-3019 } & \text { Anti-CTGF antibody } \\ \text { NCT01362231 } & \text { AB0024 } & \text { Anti-LOXL2 monoclonal antibody } \\ \text { NCT01385644 } & \text { MSCs } & \text { Epithelial tissue repair }\end{array}$

MSC: mesenchymal stem cell; IL: interleukin; CCL: chemokine (C-C motif) ligand; CTGF: connective tissue growth factor; LOXL: lysyl oxidase-like. \#: identifier numbers are provided for http://clinicaltrials.gov/ 
Mesenchymal stem cells (MSCs) are one of the most intriguing novel therapeutic approaches in the field of chronic diseases [75]. In animal models of bleomycin-induced fibrosis, which do not represent the progressive and lethal nature of IPF, it has been shown that systematically administered bone marrow (BM)-MSCs can differentiate into type II epithelial cells and suppress expression of proinflammatory and profibrotic genes [75]. Recently it has been shown that BM-MCSs from patients with IPF and rheumatoid arthritis-UIP present relative telomere lengths which do not differ from healthy controls and, thus, could be used for cell replacement treatment [76]. These cells can migrate to the site of lung injury through a C-X-C chemokine receptor type 4 (CXCR4)-dependent mechanism [77]. BM-MSCs have been tested in clinical trials for diseases other than IPF, such as ischaemic heart disease, graft versus host disease, chronic obstructive pulmonary disease and refractory lupus, with encouraging results [78]. In IPF there is currently one phase I, open-label, single centre, non-randomised dose-escalation trial underway to evaluate the safety and feasibility of MSC treatment (NCT01385644).

\title{
Treatment of IPF: from 2000 to 2011 and beyond
}

In the $2000 \mathrm{ATS} / E R S$ guidelines for the management of IPF it was recommended that, if treatment is to be initiated, a combination of corticosteroids with a cytotoxic agent (azathioprine or cyclophosphamide) should be preferred over corticosteroids alone [36]. This recommendation was based mainly on expert opinion, along with a few small and not properly designed clinical trials. Since then, our understanding of the clinical and molecular characteristics of IPF have led to the development of trials targeting specific pathways and consequently the need for new recommendations, this time evidence-based [1]. According to the 2011 guidelines there is no proven pharmacologic treatment for IPF. Pharmacological treatment options have received recommendations which vary from a "strong no" to a "weak no" (table 3). However, based on recent evidence emerging from randomised controlled trials published after 2011, treatment guidelines for IPF need to be cautiously reassessed, because several weak recommendations are now considered to be strong, either against or in favour of certain pharmaceutical agents. The recommendation for the use of the combination of prednisone, azathioprine and NAC, as well as for the use of warfarin, was a "weak no" (i.e. against the use of these treatments for most patients) and should be reconsidered based on the negative results of the PANTHER-IPF [21] and ACE-IPF [24] trials, respectively. The "weak no" recommendation for the use of pirfenidone also needs reassessment in the light of the results from three phase III trials mentioned earlier, whereas the recommendation for monotherapy with NAC remains pending until the results of the continuing PANTHER-IPF trial are published. The "weak no" recommendation for treatment of pulmonary hypertension in IPF should be updated and made more specific, based on the encouraging results regarding the use of sildenafil and on the disappointing results on the use of bosentan, macitentan and ambrisentan. In the hope that the encouraging results of the phase II trial for BIBF 1120 will be coupled with positive results from the phase III trials, and following the publication of studies regarding the role of gastro-oesophageal reflux in IPF $[10,79,80]$ and the need of prospective studies to investigate it, these treatment approaches will surely need to be considered in the future.

Clinicians should always keep in mind that palliative care is equally important, mainly in patients with advanced disease. The use of diamorphine can reduce anxiety, dyspnoea and cough without causing a

\section{TABLE 3 Pharmacological treatments in idiopathic pulmonary fibrosis (IPF)}

\author{
Strong no recommendation \\ Corticosteroid monotherapy \\ Colchicine \\ Cyclosporin A \\ A combination of corticosteroids and immunomodulatory therapy \\ Interferon- $\gamma-1 b$ \\ Bosentan \\ Etanercept \\ Weak yes recommendation \\ Corticosteroids in patients with acute exacerbation \\ Treatment of asymptomatic gastro-oesophageal reflux \\ Weak no recommendation \\ Combination of prednisone, azathioprine and NAC: needs to be updated \\ Monotherapy with NAC: further update pending \\ Pirfenidone: needs to be updated \\ Anticoagulants: needs to be updated \\ $\mathrm{PH}$ associated with IPF: needs to be updated
}

NAC: $\mathrm{N}$-acetyl-cysteine; $\mathrm{PH}$ : pulmonary hypertension. 
significant decrease in oxygen saturation [81]. Thalidomide has been proven to be effective in the treatment of cough [82]. Corticosteroids have also been tested in the treatment of cough [83], but their use is not recommended in light of the results of recent trials mentioned above.

\section{Design of clinical trials}

Since 2004, when the first appropriately designed clinical trial in IPF was published, there have been a continuously growing number of trials, each using one of various parameters as the primary end-point. The debate on which is the most clinically meaningful end-point is ongoing. Recently, it has been proposed that all-cause mortality and all-cause hospitalisation are the most appropriate end-points as they can directly measure symptoms, functional status and survival of IPF patients [84]. However, there are some limitations that should be discussed [85]. Clinical trials adequate for evaluation of mortality as an end-point should include an extremely high number of patients and require a much longer follow-up time in order for the results to reach statistical significance. As consequence, the costs will be much higher preventing pharmaceutical companies from investing and developing new drugs for IPF. In addition, it seems obvious that patients will not agree to stay in a trial for a long time, in either the placebo or the treatment arm, if their disease progresses and they will seek to enrol in other ongoing trials. It is well known that IPF is a very complex disease with a lot of comorbidities, which may be the cause of hospitalisation and mortality, which are not expected to be directly influenced by novel agents with antifibrotic properties. For these reasons a surrogate marker for mortality is needed and serial changes in FVC seem to be the preferred option. Changes in absolute FVC $>10 \%$ over a period of 6 or 12 months are considered a surrogate marker for mortality and provide evidence of, in the absence of an alternative explanation, disease progression [1]. However, it is still uncertain what the optimal threshold for a significant change is, as a change of $5-10 \%$ is also associated with disease progression [86]. Recently, it has been proposed that progression-free survival could be a clinically meaningful end-point [2]. It has been successfully used in clinical trials of nonsmall cell lung cancer in which combinations of therapeutic agents have been investigated [87]. Sufficient similarities in the biology and clinical behaviour of lung cancer and IPF [2] suggest that the same strategy can be applied in clinical trials for IPF.

Another issue that should be noted is that not all the patients with IPF recruited in a trial will have similar responses to the same pharmacological agent or similar disease behaviour. The disease is characterised by clinical heterogeneity with patients that progress slowly or rapidly, patients can also develop disproportionate pulmonary hypertension, concurrent emphysema or lung cancer. Hence, what is warranted is a marker able to predict the longitudinal behaviour of the disease, in order to include patients with same disease behaviour in future trials. Currently patients with probable or possible IPF [1] are not included in ongoing trials and whether these patients should be recruited in future trials is still under discussion.

In addition, as various pathways are simultaneously activated in IPF it is utopic to believe that using an agent that blocks a single pathway would be enough to slow disease progression. In the field of other respiratory diseases, such as lung cancer, tuberculosis, COPD and asthma, the current treatment recommendation includes a combination of agents with different mechanisms of action. We believe that the same strategy should be applied to IPF. Therefore, clinical studies of combination therapies are urgently needed.

\section{Conclusion}

During the past decade a lot of progress has been made in the basic science underlying the pathogenesis of IPF. Consequently, trials targeting different pathways have been developed, leading to licensing of the first IPF-specific drug, pirfenidone. The main aim for the future is to optimise the design of clinical trials by selecting clinically meaningful end-points and patients who are more likely to respond to the agent under investigation, and by referring patients to specialist IPF centres to be recruited into novel trials.

\section{References}

Raghu G, Collard HR, Egan JJ, et al. An official ATS/ERS/JRS/ALAT statement: idiopathic pulmonary fibrosis: evidence-based guidelines for diagnosis and management. Am J Respir Crit Care Med 2011; 183: 788-824.

2 Vancheri C, du Bois RM. A progression-free end-point for idiopathic pulmonary fibrosis trials: lessons from cancer. Eur Respir J 2013; 41: 262-269.

3 Nalysnyk L, Cid-Ruzafa J, Rotella P, et al. Incidence and prevalence of idiopathic pulmonary fibrosis: review of the literature. Eur Respir Rev 2012; 21: 355-361.

4 du Bois RM. An earlier and more confident diagnosis of idiopathic pulmonary fibrosis. Eur Respir Rev 2012; 21: $141-146$.

5 Margaritopoulos GA, Romagnoli M, Poletti V, et al. Recent advances in the pathogenesis and clinical evaluation of pulmonary fibrosis. Eur Respir Rev 2012; 21: 48-56.

6 Wuyts WA, Agostini C, Antoniou KM, et al. The pathogenesis of pulmonary fibrosis: a moving target. Eur Respir J 2013; 41: 1207-1218. 
7 Günther A, Korfei M, Mahavadi P, et al. Unravelling the progressive pathophysiology of idiopathic pulmonary fibrosis. Eur Respir Rev 2012; 21: 152-160.

8 Selman M, King TE, Pardo A. Idiopathic pulmonary fibrosis: prevailing and evolving hypotheses about its pathogenesis and implications for therapy. Ann Intern Med 2001; 134: 136-151.

9 Lasithiotaki I, Antoniou KM, Vlahava VM, et al. Detection of herpes simplex virus type-1 in patients with fibrotic lung diseases. PLoS One 2011; 6: e27800.

10 Tcherakian C, Cottin V, Brillet PY, et al. Progression of idiopathic pulmonary fibrosis: lessons from asymmetrical disease. Thorax 2011; 66: 226-231.

11 Lee JS, Ryu JH, Elicker BM, et al. Gastroesophageal reflux therapy is associated with longer survival in idiopathic pulmonary fibrosis. Am J Respir Crit Care Med 2011; 184: 1390-1394.

12 Samara KD, Margaritopoulos G, Wells AU, et al. Smoking and pulmonary fibrosis: novel insights. Pulm Med 2011; 2011: 461439.

13 Maher TM, Wells AU, Laurent GJ. Idiopathic pulmonary fibrosis: multiple causes and multiple mechanisms? Eur Respir J 2007; 30: 835-839.

14 Armanios M. Syndromes of telomere shortening. Annu Rev Genomics Hum Genet 2009; 10: 45-61.

15 Coppé JP, Desprez PY, Krtolica A, et al. The senescence-associated secretory phenotype: the dark side of tumor suppression. Annu Rev Pathol 2010; 5: 99-118.

16 Chilosi M, Doglioni C, Murer B, et al. Epithelial stem cell exhaustion in the pathogenesis of idiopathic pulmonary fibrosis. Sarcoidosis Vasc Diffuse Lung Dis 2010; 27: 7-18.

17 Korfei M, Ruppert C, Mahavadi P, et al. Epithelial endoplasmic reticulum stress and apoptosis in sporadic idiopathic pulmonary fibrosis. Am J Respir Crit Care Med 2008; 178: 838-846.

18 Lawson WE, Cheng DS, Degryse AL, et al. Endoplasmic reticulum stress enhances fibrotic remodeling in the lungs. Proc Natl Acad Sci USA 2011; 108: 10562-10567.

19 Sisson TH, Mendez M, Choi K, et al. Targeted injury of type II alveolar epithelial cells induces pulmonary fibrosis. Am J Respir Crit Care Med 2010; 181: 254-263.

20 Demedts M, Behr J, Buhl R, et al. High-dose acetylcysteine in idiopathic pulmonary fibrosis. N Engl J Med 2005; 353: 2229-2242.

21 Idiopathic Pulmonary Fibrosis Clinical Research Network, Raghu G, Anstrom KJ, et al. Prednisone, azathioprine, and N-acetylcysteine for pulmonary fibrosis. N Engl J Med 2012; 366: 1968-1977.

22 Taniguchi H, Ebina M, Kondoh Y, et al. Pirfenidone in idiopathic pulmonary fibrosis. Eur Respir J 2010; 35: 821-829.

23 Noble PW, Albera C, Bradford WZ, et al. Pirfenidone in patients with idiopathic pulmonary fibrosis (CAPACITY): two randomised trials. Lancet 2011; 377: 1760-1769.

24 Noth I, Anstrom KJ, Calvert SB, et al. A placebo-controlled randomized trial of warfarin in idiopathic pulmonary fibrosis. Am J Respir Crit Care Med 2012; 186: 88-95.

25 Richeldi L, Costabel U, Selman M, et al. Efficacy of a tyrosine kinase inhibitor in idiopathic pulmonary fibrosis. N Engl J Med 2011; 365: 1079-1087.

26 Daniels CE, Lasky JA, Limper AH, et al. Imatinib treatment for idiopathic pulmonary fibrosis: randomized placebocontrolled trial results. Am J Respir Crit Care Med 2010; 181: 604-610.

27 Zisman DA, Schwarz M, Anstrom KJ, et al. A controlled trial of sildenafil in advanced idiopathic pulmonary fibrosis. N Engl J Med 2010; 363: 620-628.

28 King TE, Behr J, Brown KK, et al. BUILD-1: a randomized placebo-controlled trial of bosentan in idiopathic pulmonary fibrosis. Am J Respir Crit Care Med 2008; 177: 75-81.

29 King TE, Brown KK, Raghu G, et al. BUILD-3: a randomized, controlled trial of bosentan in idiopathic pulmonary fibrosis. Am J Respir Crit Care Med 2011; 184: 92-99.

30 Raghu G, Behr J, Brown K, et al. Artemis-IPF: a placebo-controlled trial of ambrisentan in idiopathic pulmonary fibrosis. B93. Clinical Trials in Idiopathic Pulmonary Fibrosis and Sarcoidosis. May 1, 2012; A3632.

31 Raghu G, Million-Rousseau R, Morganti A, et al. Efficacy and safety of macitentan in idiopathic pulmonary fibrosis: results of a prospective, randomized, double-blind, placebo-controlled trial. B93. Clinical Trials in Idiopathic Pulmonary Fibrosis and Sarcoidosis. May 1, 2012; A3631.

32 Raghu G, Brown KK, Bradford WZ, et al. A placebo-controlled trial of interferon- $\gamma-1 \mathrm{~b}$ in patients with idiopathic pulmonary fibrosis. $N$ Engl J Med 2004; 350: 125-133.

33 King TE Jr, Albera C, Bradford WZ, et al. Effect of interferon- $\gamma-1 \mathrm{~b}$ on survival in patients with idiopathic pulmonary fibrosis (INSPIRE): a multicentre, randomised, placebo-controlled trial. Lancet 2009; 374: 222-228.

34 du Bois RM. Idiopathic pulmonary fibrosis: present understanding and future options. Eur Respir Rev 2011; 20: 132-133.

35 Cottin V. Changing the idiopathic pulmonary fibrosis treatment approach and improving patient outcomes. Eur Respir Rev 2012; 21: 161-167.

36 American Thoracic Society. Idiopathic pulmonary fibrosis: diagnosis and treatment. International consensus statement. American Thoracic Society (ATS), and the European Respiratory Society (ERS). Am J Respir Crit Care Med 2000; 161: 646-664.

37 Raghu G, Depaso WJ, Cain K, et al. Azathioprine combined with prednisone in the treatment of idiopathic pulmonary fibrosis: a prospective double-blind, randomized, placebo-controlled clinical trial. Am Rev Respir Dis 1991; 144: 291-296.

38 Richeldi L. Assessing the treatment effect from multiple trials in idiopathic pulmonary fibrosis. Eur Respir Rev 2012; 21: $147-151$

39 Spagnolo P, Del Giovane C, Luppi F, et al. Non-steroid agents for idiopathic pulmonary fibrosis. Cochrane Database Syst Rev 2010; 9: CD003134.

40 Behr J, Maier K, Degenkolb B, et al. Antioxidative and clinical effects of high-dose N-acetylcysteine in fibrosing alveolitis. Adjunctive therapy to maintenance immunosuppression. Am J Respir Crit Care Med 1997; 156: 1897-1901.

41 Behr J, Degenkolb B, Krombach F, et al. Intracellular glutathione and bronchoalveolar cells in fibrosing alveolitis: effects of N-acetylcysteine. Eur Respir J 2002; 19: 906-911.

42 NIH News. Commonly used three-drug regimen for idiopathic pulmonary fibrosis found harmful. Bethesda, National Institutes of Health, October 21, 2011. www.nih.gov/news/health/oct2011/nhlbi-21.htm 
Wells AU, Behr J, Costabel U, et al. Triple therapy in idiopathic pulmonary fibrosis: an alarming press release. Eur Respir J 2012; 39: 805-806.

44 Maher TM. Pirfenidone in idiopathic pulmonary fibrosis. Drugs Today (Barc) 2010; 46: 473-482.

45 Schaefer CJ, Ruhrmund DW, Pan L, et al. Antifibrotic activities of pirfenidone in animal models. Eur Respir Rev 2011; 20: 85-97.

46 Iyer SN, Gurujeyalakshmi G, Giri SN. Effects of pirfenidone on transforming growth factor- $\beta$ gene expression at the transcriptional level in bleomycin hamster model of lung fibrosis. J Pharmacol Exp Ther 1999; 291: 367-373.

47 Gurujeyalakshmi G, Hollinger MA, Giri SN. Pirfenidone inhibits PDGF isoforms in bleomycin hamster model of lung fibrosis at the translational level. Am I Physiol 1999; 276: L311-L318.

48 Iyer SN, Gurujeyalakshmi G, Giri SN. Effects of pirfenidone on procollagen gene expression at the transcriptional level in bleomycin hamster model of lung fibrosis. J Pharmacol Exp Ther 1999; 289: 211-218.

49 Oku H, Shimizu T, Kawabata T, et al. Antifibrotic action of pirfenidone and prednisolone: different effects on pulmonary cytokines and growth factors in bleomycin-induced murine pulmonary fibrosis. Eur J Pharmacol 2008; 590: 400-408.

50 Azuma A, Nukiwa T, Tsuboi E, et al. Double-blind, placebo-controlled trial of pirfenidone in patients with idiopathic pulmonary fibrosis. Am J Respir Crit Care Med 2005; 171: 1040-1047.

51 Costabel U, Albera C, Cohen A, et al. The long-term safety of pirfenidone in patients with idiopathic pulmonary fibrosis (IPF): Interim data from the RECAP extension study. Eur Respir J 2011; 38: Suppl. 55, A174.

52 Chambers RC. Procoagulant signalling mechanisms in lung inflammation and fibrosis: novel opportunities for pharmacological intervention? Br J Pharmacol 2008; 153: Suppl. 1, S367-S378.

53 Scotton CJ, Krupiczojc MA, Königshoff M, et al. Increased local expression of coagulation factor X contributes to the fibrotic response in human and murine lung injury. J Clin Invest 2009; 119: 2550-2563.

54 Wygrecka M, Kwapiszewska G, Jablonska E, et al. Role of protease-activated receptor-2 in idiopathic pulmonary fibrosis. Am J Respir Crit Care Med 2011; 183: 1703-1714.

55 Sode BF, Dahl M, Nielsen SF, et al. Venous thromboembolism and risk of idiopathic interstitial pneumonia: a nationwide study. Am J Respir Crit Care Med 2010; 181: 1085-1092.

56 Kubo H, Nakayama K, Yanai M, et al. Anticoagulant therapy for idiopathic pulmonary fibrosis. Chest 2005; 128: 1475-1482.

57 Tzouvelekis A, Margaritopoulos G, Loukides S, et al. Warfarin in idiopathic pulmonary fibrosis: friend or foe, is it a matter of genes and heparin? Am J Respir Crit Care Med 2013; 187: 213-214.

58 Allen JT, Spiteri MA. Growth factors in idiopathic pulmonary fibrosis: relative roles. Respir Res 2002; 3: 13.

59 Daniels CE, Wilkes MC, Edens M, et al. Imatinib mesylate inhibits the profibrogenic activity of TGF- $\beta$ and prevents bleomycin-mediated lung fibrosis. J Clin Invest 2004; 114: 1308-1316.

60 Aono Y, Nishioka Y, Inayama M, et al. Imatinib as a novel antifibrotic agent in bleomycin-induced pulmonary fibrosis in mice. Am J Respir Crit Care Med 2005; 171: 1279-1285.

61 Vuorinen K, Gao F, Oury TD, et al. Imatinib mesylate inhibits fibrogenesis in asbestos-induced interstitial pneumonia. Exp Lung Res 2007; 33: 357-373.

62 Clark JG, Dedon TF, Wayner EA, et al. Effects of interferon- $\gamma$ on expression of cell surface receptors for collagen and deposition of newly synthesized collagen by cultured human lung fibroblasts. J Clin Invest 1989; 83: 1505-1511.

63 Narayanan AS, Whithey J, Souza A, et al. Effect of gamma-interferon on collagen synthesis by normal and fibrotic human lung fibroblasts. Chest 1992; 101: 1326-1331.

64 Okada T, Sugie I, Aisaka K. Effects of gamma-interferon on collagen and histamine content in bleomycin-induced lung fibrosis in rats. Lymphokine Cytokine Res 1993; 12: 87-91.

65 Gurujeyalakshmi G, Giri SN. Molecular mechanisms of antifibrotic effect of interferon- $\gamma$ in bleomycin-mouse model of lung fibrosis: downregulation of TGF- $\beta$ and procollagen I and III gene expression. Exp Lung Res 1995; 21 : 791-808.

66 Ziesche R, Hofbauer E, Wittmann K, et al. A preliminary study of long-term treatment with interferon- $\gamma-1 \mathrm{~b}$ and low-dose prednisolone in patients with idiopathic pulmonary fibrosis. N Engl J Med 1999; 341: 1264-1269.

67 Raghu G, Brown KK, Costabel U, et al. Treatment of idiopathic pulmonary fibrosis with etanercept: an exploratory, placebo-controlled trial. Am J Respir Crit Care Med 2008; 178: 948-955.

68 Hancock A, Armstrong L, Gama R, et al. Production of interleukin 13 by alveolar macrophages from normal and fibrotic lung. Am J Respir Cell Mol Biol 1998; 18: 60-65.

69 Moore BB, Kolodsick JE, Thannickal VJ, et al. CCR2-mediated recruitment of fibrocytes to the alveolar space after fibrotic injury. Am J Pathol 2005; 166: 675-684.

70 Murray LA, Argentieri RL, Farrell FX, et al. Hyper-responsiveness of IPF/UIP fibroblasts: interplay between TGF $\beta 1$, IL-13 and CCL2. Int J Biochem Cell Biol 2008; 40: 2174-2182.

71 Lipson KE, Wong C, Teng Y, et al. CTGF is a central mediator of tissue remodeling and fibrosis and its inhibition can reverse the process of fibrosis. Fibrogenesis Tissue Repair 2012; 5: Suppl. 1, S24.

72 Akiri G, Sabo E, Dafni H, et al. Lysyl oxidase-related protein-1 promotes tumor fibrosis and tumor progression in vivo. Cancer Res 2003; 63: 1657-1666.

73 Barry-Hamilton V, Spangler R, Marshall D, et al. Allosteric inhibition of lysyl oxidase-like-2 impedes the development of a pathologic microenvironment. Nat Med 2010; 16: 1009-1017.

74 Vadasz Z, Kessler O, Akiri G, et al. Abnormal deposition of collagen around hepatocytes in Wilson's disease is associated with hepatocyte specific expression of lysyl oxidase and lysyl oxidase like protein-2. J Hepatol 2005; 43: 499-507.

75 Tzouvelekis A, Antoniadis A, Bouros D. Stem cell therapy in pulmonary fibrosis. Curr Opin Pulm Med 2011; 17: 368-373.

76 Antoniou KM, Margaritopoulos GA, Proklou A, et al. Investigation of telomerase/telomeres system in bone marrow mesenchymal stem cells derived from IPF and RA-UIP. I Inflamm (Lond) 2012; 9: 27.

77 Antoniou KM, Papadaki HA, Soufla G, et al. Investigation of bone marrow mesenchymal stem cells (BM MSCs) involvement in idiopathic pulmonary fibrosis (IPF). Respir Med 2010; 104: 1535-1542.

78 Toonkel RL, Hare JM, Matthay MA, et al. Mesenchymal stem cells and idiopathic pulmonary fibrosis: potential for clinical testing. Am J Respir Crit Care Med 2013 [In press DOI:10.1164/rccm.201207-1204PP]. 
79 Noth I, Zangan SM, Soares RV, et al. Prevalence of hiatal hernia by blinded multidetector CT in patients with idiopathic pulmonary fibrosis. Eur Respir J 2012; 39: 344-351.

80 Lee JS, Song JW, Wolters PJ, et al. Bronchoalveolar lavage pepsin in acute exacerbation of idiopathic pulmonary fibrosis. Eur Respir J 2012; 39: 352-358.

81 Allen S, Raut S, Woollard J, et al. Low dose diamorphine reduces breathlessness without causing a fall in oxygen saturation in elderly patients with end-stage idiopathic pulmonary fibrosis. Palliat Med 2005; 19: 128-130.

82 Horton MR, Santopietro V, Mathew L, et al. Thalidomide for the treatment of cough in idiopathic pulmonary fibrosis: a randomized trial. Ann Intern Med 2012; 157: 398-406.

83 Hope-Gill BDM, Hilldrup S, Davies C, et al. A study of the cough reflex in idiopathic pulmonary fibrosis. Am J Respir Crit Care Med 2003; 168: 995-1002.

84 Raghu G, Collard HR, Anstrom KJ, et al. Idiopathic pulmonary fibrosis: clinically meaningful primary endpoints in phase 3 clinical trials. Am J Respir Crit Care Med 2012; 185: 1044-1048.

85 Wells AU, Behr J, Costabel U, et al. Hot of the breath: mortality as a primary end-point in IPF treatment trials: the best is the enemy of the good. Thorax 2012; 67: 938-940.

86 Zappala CJ, Latsi PI, Nicholson AG, et al. Marginal decline in forced vital capacity is associated with a poor outcome in idiopathic pulmonary fibrosis. Eur Respir J 2010; 35: 830-836.

87 Bria E, Gralla RJ, Raftopoulos H, et al. Magnitude of benefit of adjuvant chemotherapy for non-small cell lung cancer: meta-analysis of randomized clinical trials. Lung Cancer 2009; 63: 50-57. 